\title{
Clinical and endoscopic spectrum of upper gastrointestinal manifestations in HIV patients
}

Ravikumar VN', Rudresh $K^{2}$, Jalihal $\mathbf{U}^{3}$, Satish $\mathbf{R}^{4}$, Manjunath $\mathbf{R}^{5}$

${ }^{1}$ Assistant Professor, ${ }^{2}$ Professor, ${ }^{3}$ Associate Professor, ${ }^{4}$ Resident, ${ }^{5}$ Post Graduate, Department of Medicine, M.S. Ramaiah Medical College, Bangalore, India

\begin{abstract}
Background: Human Immunodeficiency Virus (HIV) infected patient frequently report upper gastrointestinal (GI) symptoms; however their prevalence and diagnostic approach is not well known.

Objective: The objective of this study was to study clinical, endoscopic and histopathological changes in HIV infected patients with upper GI symptoms and their correlation with CD4 count.

Materials and methods: We evaluated $50 \mathrm{HIV}$ infected patients who presented to M.S. Ramaiah hospital with upper GI symptoms. All patients answered questionnaire assessing upper GI symptoms and underwent upper GI endoscopy. Mucosal biopsy was taken wherever mucosal abnormality seen.

Results: In our study, the mean age of patients was 40.98 yrs, of which $80 \%$ were males. Vomiting (36\%), epigastric pain (36\%), weight loss (34\%) and anorexia (34\%) were the predominant symptoms. Esophagogastroduodenoscopy (EGD) findings revealed- Oesophageal candidiasis in $28.0 \%$, esophagitis in $22.0 \%$, gastritis in $20.0 \%$, duodenitis in $14 \%$, normal upper GI mucosa in $18 \%$ patients. Oesophageal candidiasis was the most common finding on histopathological examination and the mean CD4 count was 157.92 cells $/ \mu 1$.

Conclusion: Vomiting, epigastric pain, weight loss and anorexia were most frequent symptoms. Oral candidiasis was the most common oral lesion. Oesophageal candidiasis, oesophagitis and oesophageal ulcers were the common findings on EGD. Patient with CD4 count less than 200cells/ $\mu 1$ had more frequent upper GI mucosal involvement than in patients with CD4 count more than 200. Majority of the patients with GI symptoms had upper GI mucosal changes and opportunistic infections. Thus endoscopic and histopathological evaluation is advisable for the early diagnosis and treatment of upper GI complications in patients with HIV infection.
\end{abstract}

Key words: AIDS, Oesophageal candidiasis, Esophagogastroduodenoscopy, HIV, Upper gastrointestinal symptoms.

$\mathrm{T}$ he spectrum of the HIV infection varies from asymptomatic infection to acquired immunodeficiency syndrome (AIDS). The GI tract has long been recognised as a major site of HIV related diseases and up to $90 \%$ of HIV infected patients experience GI symptoms during the course of their disease. However, the exact prevalence and diagnostic approach in these patients is not clearly defined ${ }^{1}$. HIV infection leads to progressive deterioration of both the general and the local immune defence system of the GI tract mucosa $a^{2,3}$. Opportunistic infections are the most frequent GI complications of HIV infection and remain a major cause of morbidity and mortality in these patients $^{2,4}$.

These diseases account for high prevalence of upper GI symptoms such as dysphagia, odynophagia, abdominal pain $^{5,6}$. With the progression of immunodeficiency, EGD becomes a useful diagnostic modality for the early diagnosis of these opportunistic infections and other inflammatory conditions $s^{7,8}$. Hence we studied the clinical, endoscopic and biopsy changes in HIV patients with upper GI symptoms.

\section{Materials and methods}

This prospective observational study was undertaken in 50 HIV infected patients with upper GI symptoms presenting to M.S.Ramaiah hospital to evaluate clinical, endoscopic and histopathologic changes in HIV infected patients with upper gastro intestinal symptoms and to correlate with CD 4 count. All HIV infected patients above 18 yrs presenting with upper GI symptoms from november 2007 to october 2009 were included. HIV patients with other associated immunocompromised

Correspondence

Dr. Ravikumar.V.N

Assistant Professor

M.S. Ramaiah Medical College

Bangalore, India

E-mail: dr.rkvn@gmail.com 
states, chronic liver disease, chronic renal failure, steroid therapy, chronic NSAIDs and antifungal therapy were excluded as they may be confounding factors for upper GI symptoms. All patients answered a questionnaire and were subjected to physical examination followed by investigations (complete blood count, renal function tests, liver function tests, Urine routine, Chest $\mathrm{x}$ ray, ELISA for HIV 1 and 2, Western Blot and CD4 count). Swabs were collected in cases presenting with oral / oropharyngeal thrush.

All patients were subjected for upper GI endoscopy after taking prior consent. Premedication was given with Xylocaine mouth wash and Midazolam 2mg intravenous injection. Pentax EG 290 P video endoscope was used to visualise the upper GI tract. It was disinfected with $2 \%$ glutaraldehyde before and after the procedure. Oesophageal, gastric and duodenal mucosa was carefully examined for evidence of inflammation, ulceration, erosions, oedema, hemorrhagic patches and opportunistic infection. Mucosal biopsy, followed by histopathological examination was done if mucosal abnormality was found. Biopsy specimens were immediately fixed in buffered formalin and submitted for histopathologic study. Routine histological evaluation (haematoxylin and eosin; H\&E), immunohistochemistry staining for CMV and HSV antigens and staining for mycobacterium, fungi (Gomori-methenamine-silver nitrate and periodic acid-Schiff) and microsporidia (Gram and or trichrome stain) were performed where appropriate. Biopsy specimens for light microscopy were processed using standard techniques.

Table 1: Symptoms in hiv patients

\begin{tabular}{|l|c|c|}
\hline \multirow{2}{*}{ Symptoms } & \multicolumn{2}{|c|}{ HIV patients $(\mathbf{n}=\mathbf{5 0})$} \\
\cline { 2 - 3 } & No & \% \\
\hline 1.Vomiting & 18 & 36.0 \\
\hline 2.Epigastric pain & 18 & 36.0 \\
\hline 3.Anorexia & 17 & 34.0 \\
\hline 4. Weight loss & 17 & 34.0 \\
\hline 5.Dysphagia & 13 & 26.0 \\
\hline 6.Nausea & 11 & 22.0 \\
\hline 7. Diarrhea & 10 & 20.0 \\
\hline 8.Dyspepsia & 6 & 12.0 \\
\hline 9.Hematemesis & 5 & 10.0 \\
\hline 10. Odynophagia & 4 & 8.0 \\
\hline
\end{tabular}

The major presenting symptoms were vomiting, epigastric pain and dysphagia.

\section{Results}

We studied 50 HIV infected patients with upper GI symptoms. The mean age in years was 40.98. Majority were males, who constituted $80 \%$ and females $20 \%$. Thirty $\operatorname{six}(72.0 \%)$ patients were not on anti retroviral therapy. The major presenting symptoms were vomiting and epigastric pain, which constituted $36.0 \%$ each, followed by dysphagia in $26.0 \%$, nausea in $22 \%$ (Table 1).

Oral candidiasis was seen in $44.0 \%$ of patients, oral ulcers in $14.0 \%$, and chelitis in $4.0 \%$. 15 (30.0\%) patients had CD4 count less than 100, 20 patients (40.0\%) had between 101 to 200 and 15 patients $(30.0$ \%) more than 200. Mean CD4 count was 157.92/ $\mu 1$. Majority of patients were in WHO clinical stage IV $(42.0 \%)$, followed by $32.0 \%$ in stage III, $22.0 \%$ in stage II, $4.0 \%$ in stage I of the disease.

EGD findings revealed oesophageal candidiasis in 14 patients $(28.0 \%)$, oesophagitis in $22.0 \%$, gastritis in $20.0 \%$, duodenitis in $14 \%$, normal upper GI mucosa in $18 \%$ patients (Table2). Histopathological examination revealed oesophageal candidiasis in $39.02 \%$, acute oesophagitis in $21.95 \%$ and CMV oesophagitis in $4.87 \%$, acute gastritis in $19.5 \%$, chronic gastritis in $4.87 \%$, acute duodenitis in $4.87 \%$, and chronic duodenitis in $12.19 \%$ ( Table3). Oesophageal candidiasis, oesophagitis, oesophageal ulcers, gastritis and duodenitis were more commonly associated with the CD4 count $<200$ (Table4).

Table 2: Esophago-Gastro-Duodenoscopy findings

\begin{tabular}{|l|c|c|}
\hline \multirow{2}{*}{ Endoscopy findings } & \multicolumn{2}{|c|}{ HIV patients (n=50) } \\
\cline { 2 - 3 } & No & \% \\
\hline Esophagus & & \\
\hline Candidiasis & 14 & 28 \\
\hline Esophagitis & 11 & 22 \\
\hline Ulcers & 8 & 16 \\
\hline Stomach & 10 & 20 \\
\hline Gastritis & 2 & 4 \\
\hline Congestive gastropathy & 7 & 14 \\
\hline $\begin{array}{l}\text { Duodenum } \\
\text { Duodenitis }\end{array}$ & 9 & 18 \\
\hline $\begin{array}{l}\text { Normal Upper GI } \\
\text { Mucosa }\end{array}$ & & \\
\hline
\end{tabular}

Candidiasis, esophagitis and gastritis were the common findings on esophago-gastro-duodenoscopy. 
Table 3: Histopathological findings

\begin{tabular}{|l|c|c|}
\hline \multirow{2}{*}{ Histopathological findings } & \multicolumn{2}{|c|}{ HIV patients (n=41) } \\
\cline { 2 - 3 } & No & \% \\
\hline Esophagus & & \\
\hline Esophageal candidiasis & 16 & 39.02 \\
\hline Acute esophagitis & 9 & 21.95 \\
\hline CMV esophagitis & 2 & 4.87 \\
\hline Stomach & & \\
\hline Acute gastritis & 8 & 19.5 \\
\hline Chronic gastritis & 2 & 4.87 \\
\hline Duodenum & & \\
\hline Chronic duodenitis & 5 & 12.19 \\
\hline Acute Duodenitis & 2 & 4.87 \\
\hline
\end{tabular}

Histopathological examination revealed esophageal candidiasis, acute esophagitis, and acute gastritis in majority of cases.

\section{Discussion}

Gastrointestinal manifestations are among the most frequent complaints in patients with HIV infection. The reported incidence of GI manifestation in the literature varied from $50 \%$ to $93 \%$, Majority of the patients presented with vomiting, epigastric pain and dyspepsia. Oral candidiasis, oesophageal candidiasis and oesophagitis were most commonly seen lesions. 14 out of 50 patients were on ART and all of them were on Zidovudine, Lamivudine and Nevirapine. Among patients receiving ART, 7 had oesophagitis, 4 had oesophageal ulcer and one had normal upper GI mucosa. ART drugs are known to cause oesophageal ulceration. Therefore, patients should be advised to take medication after food and in upright position to decrease the irritant effect. Symptoms and signs alone rarely suggest a specific aetiology or severity. Much of the frustration of managing GI disease results from confusing diagnostic process. Usage of symptom frequency and severity of symptoms correlate poorly with abnormalities on EGD. Study conducted by Corley D.A, Cello J.P, Koch J, evaluated the clinical predictors of abnormal endoscopic findings and utility of EGD for diagnosis in patients infected with HIV. They concluded that neither there were any independent symptoms predicting treatable disease on EGD nor there was any significance between severity of symptoms and EGD changes ${ }^{5}$.

The evaluation of specific gastrointestinal complaints must be based on an assessment of the degree of immunosuppression. Progressive immunocompromised state is associated with increasing prevalence of GI symptoms ${ }^{10}$. Endoscopic evaluation of the GI tract remains a cornerstone of diagnosis, especially in
Table 4: Relationship between CD4 counts and GI findings

\begin{tabular}{|l|c|c|}
\hline \multirow{2}{*}{ GI findings } & \multicolumn{2}{|c|}{ CD4 counts } \\
\cline { 2 - 3 } & $\begin{array}{c}<\mathbf{2 0 0} \\
(\mathbf{n = 3 5})\end{array}$ & $\begin{array}{c}\mathbf{2 0 0} \\
(\mathbf{n}=\mathbf{1 5})\end{array}$ \\
\hline Esophageal candidiasis & $12(34.28 \%)$ & $2(13.34 \%)$ \\
Esophagitis & $9(25.71 \%)$ & $2(13.34 \%)$ \\
Esophageal ulcers & $7(20 \%)$ & $1(6.67 \%)$ \\
Gastritis & $7(20.0 \%)$ & $3(20.6 \%)$ \\
Duodenitis & $6(17.14 \%)$ & $1(6.67 \%)$ \\
Normal & $3(8.57 \%)$ & $6(40.0 \%)$ \\
\hline
\end{tabular}

Esophageal candidiasis, esophagitis, esophageal ulcers, gastritis and duodenitis are commonly associated with the CD4 $<200$ cells $/ \mu 1$.

patients with advanced immunodeficiency, who are at risk for opportunistic infection. The CD4 lymphocyte count helps to predict the risk of an OI, with the highest risk seen in HIV-infected patients with low CD4 count $(<200 \text { cells } / \mu \mathrm{l})^{11}$. Demonstration of a pathogen in tissue is the most specific means of establishing an etiologic diagnosis in opportunistic infections ${ }^{6}$.

In our study, $70 \%$ patients were having CD4 count less than 200cells/ $\mu$, indicating patients with CD4 count less than 200cells/ $\mu$ l had more frequent upper GI mucosal involvement and opportunistic infection when compared to those with CD4 count more than 200cells/ $\mu$ l. In a study done by Olmos MA et al, upper GI endoscopy with biopsies more frequently detected opportunistic and non-opportunistic diseases in HIV infected patients with upper gastrointestinal symptoms. Opportunistic diseases were related to lower CD4 counts. Non-opportunistic diseases had similar frequency in both groups, HIV positive and negative controls ${ }^{12}$. Pathogens are not usual in normal oesophagoduodenal mucosa of HIV infected patients with dyspepsia. In oesophagus and duodenum, biopsies should only be taken in advanced immunocompromised patients ${ }^{13}$.

EGD in HIV patients can serve the following purposes: 1) Many diseases which cannot be diagnosed by routine investigations and require specific treatment can be diagnosed by EGD. 2) Prescribing empirical treatment without performing upper endoscopy might put the patients at risk of many side effects from the unnecessary medicines, for instance $\mathrm{H} 2$ blocker for abdominal pain may predispose the patient to fungal overgrowth, and 
antiviral drugs may cause various adverse effects and emergence of resistant strains. 3) EGD can be helpful in identifying AIDS defining illnesses.

Limitations of our study include 1. Relatively small study group, as large number of HIV patients were excluded in view of associated confounding factors 2.Patients treated empirically for a GI opportunistic infection without prior endoscopic evaluation could have been missed.

\section{Conclusion}

Upper-GI endoscopy identified a diverse spectrum of disease and provided information that would be clinically relevant to most $\mathrm{HIV}$-infected patients with upper GI symptoms. Majority of the patients with GI symptoms had upper GI mucosal changes and opportunistic infections, thus endoscopic and histopathological evaluation is advisable for the early diagnosis and treatment of upper GI complications in patients with HIV infection.

In the light of low CD4 count strongly correlating with upper GI symptoms and endoscopic findings of opportunistic infections in the present study, routine EGD in asymptomatic HIV infected patients with CD4 count $<200$ cells/ $\mu$ l may be useful, after validation by a larger study.

\section{References}

1. Lorenz KA, Shapir MF, Asch SM, Bozzete SA, Hays RD. Association of symptoms and health related quality of life: Findings from a National study of persons with HIV infection. Ann Intern Med. 2001; 134:854-68.

2. Wilcox CM. Gastrointestinal consequences of infection with human immunodeficiency virus. In: Feldman M, Friedman LS, Sleisenger $\mathrm{MH}$, editors. Sleisenger and Fordtran's Gastrointestinal and liver diseases. Philadelphia: Saunders; 2002.p. 487-97.

3. Ullrich R, Zeitz M, Riecken EO. Enteric immunologic abnormalities in human immunodeficiency virus infection. Semin Liver Dis.1992; 12:167-74.
4. Reznik DA. Oral Manifestations of HIV Disease. Top HIV Med. 2005; 13(5): 143-148.

5. Corley DA, Cello JP, Koch J. Evaluation of upper gastrointestinal tract symptoms in patients infected with HIV. Am J Gastroenterol.1999; 94(10):2890-6.

6. Wilcox.M.C. Endoscopy in AIDS: "A pattern approach to diagnosis".VHJOE. 2005;4:1-7.

7. Wilcox CM. Role of endoscopy in the investigation of upper gastrointestinal symptoms in HIV-infected patients. Can J Gastroenterol. 1999;13(4):305-10.

8. Carlos CA, Kiyoshi H, Brasil HA, D'Assunção MA,Daniel M, Armellini S et al. Role of upper gastrointestinal endoscopy with routine standardized endoscopic biopsy in AIDS. Dig Endosc. 2002; 14(4):152-5.

9. Gupta A, Changra M, Agarwal C.G, Mishra R, Tripati AK, NAG VL. Gastrointestinal Manifestation of HIV Disease. J Assoc Physicians India. 2005; 53: 376.

10. May GR, Gill MJ, Church DL et al. Gastrointestinal symptoms in ambulatory HIVinfected patients. Dig Dis Sci. 1993; 138:138894.

11. Werneck-Silva AL, Prado IB. Role of upper endoscopy in diagnosing opportunistic infections in human immunodeficiency virusinfected patients World J Gastroenterol. 2009;15(9):1050-6.

12. Olmos MA, Fanín A, Araya V, Piskorz E, Quesada EC, Magnanini F et al Endoscopic approach in HIV infected-patients with upper gastrointestinal symptoms.Acta Gastroenterol Latinoam. 2004;34(3):120-6.

13. Werneck-Silva AL. Gastroduodenal Biopsies in Normal Mucosa of HIV Patients with Dyspepsia: Is it Worthwhile? Gastrointest Endosc. 2005; 61(5):AB158. 